\title{
Efeito da suplementação dietética com micro minerais orgânicos na produção de galinhas poedeiras
}

\section{Alexandre da Silva \\ SECHINATO ${ }^{1}$ \\ Ricardo de \\ ALBUQUERQUE ${ }^{1}$ \\ Seitiro NAKADA ${ }^{2}$}

\section{Correspondência para:}

RICARDODEALBUQUERQUE

Departamento de Nutrição e Produção

Animal

Faculdade de Medicina Veterinária e

Zootecnia

Universidade de São Paulo

Av. Duque de Caxias Norte, 225

13635-900 - Pirassununga-SP

ricalbuq@usp.br

Recebido para publicação: 05/04/2004 Aprovado para publicação: 13/02/2006

\author{
1 - Departamento de Nutrição e Produção Animal da Faculdade de \\ Medicina Veterinária e Zootecnia da Universidade de São Paulo, \\ Pirassununga - SP \\ 2 - Tortuga Companhia Zootécnica Agrária, São Paulo - SP
}

\section{Resumo}

Palavras-chave:

Um experimento foi realizado para avaliar os efeitos da suplementação dietética de fontes orgânicas de microminerais na produção de ovos de galinhas poedeiras. Foram utilizados 8 tratamentos: um com todos os minerais na forma inorgânica, um com todos na forma orgânica e mais 6 tratamentos com apenas um dos minerais na forma orgânica. Os minerais testados foram: zinco, manganês, iodo, selênio, cobre e ferro. As dietas experimentais foram à base de milho e farelo de soja. Foram utilizadas 672 aves da linhagem ISA Babcock B 300, o período de experimentação foi de 48 a 60 semanas de idade, e os parâmetros de desempenho produtivo avaliados foram: Consumo de ração, produção de ovos, massa de ovos e Conversão Alimentar. Os dados foram analisados em ciclos de postura de 28 dias. A adição dos diferentes minerais isolados ou em combinação, na forma orgânica ou inorgânica, aparentemente não resultou em melhora nas medidas de desempenho, pois à exceção do terceiro período de avaliação, quando obteve-se um menor índice de massa de ovos para o tratamento em que foi empregado o ferro orgânico $(\mathrm{p}<0,05)$, não se detectou diferença significativa. Partindo-se da premissa de que as fontes orgânicas de minerais apresentam maior biodisponibilidade, o efeito de seu uso permanece controverso, pois os resultados do experimento sugerem que a suplementação com minerais orgânicos não altera o desempenho da produção de ovos de galinhas entre 48 e 60 semanas de idade, quando comparado com a suplementação inorgânica dos mesmos.

\section{Introdução}

$\mathrm{Na}$ avicultura de postura ocorrem atualmente grandes perdas devido ao mau processamento ou má qualidade do produto, ovo. Segundo Roland ${ }^{1}$ perdas de até $15 \%$ podem ser totalizadas desde a produção até o consumidor devido a má qualidade da casca, o que está relacionado com a nutrição das aves. Para se obter uma boa nutrição é necessário que o animal receba quantidades adequadas de nutrientes, incluindose os minerais, que são considerados de grande importância para as aves, pois participam de todos os processos bioquímicos corporais. Há um interesse atual crescente em explorar fatores que aumentam a absorção ou metabolização dos elementos traços. Fontes orgânicas ou quelatos de minerais tem sido utilizadas devido a sua perspectiva de serem mais biodisponíveis.

Os minerais quelatados são definidos por Leeson e Summers ${ }^{2}$ como sendo uma mistura de elementos minerais que são ligados a algum tipo de carreador, o qual pode ser um aminoácido ou polissacarídeo, que possui a capacidade de se ligar ao metal por ligações covalentes, através de grupamentos aminos ou oxigênio, formando assim uma estrutura cíclica.

Poucos trabalhos tem sido realizados para avaliar a eficiência da suplementação mineral nas formas orgânicas ou quelatos para galinhas poedeiras em relação as formas 
inorgânicas. Segundo Reddy et al. ${ }^{3}$ as formas orgânicas aumentam a biodisponibilidade dos minerais em relação as formas inorgânicas, o que pode trazer benefícios, tais como: maior taxa de crescimento, maior ganho de peso, maior produção de ovos, melhora na qualidade de carne e ovos, redução da taxa de mortalidade e redução do efeito do estresse.

Moreng ${ }^{4}$ obteve melhora na resistência a quebra e uma significativa redução dos defeitos de casca quando as aves receberam zinco orgânico, melhora essa que não ocorreu nas aves que receberam a forma inorgânica do produto, porém, Balnave e Zhang ${ }^{5}$ em trabalho semelhante, concluiram que não houve diferença estatística na forma de suplementação de zinco para galinhas de postura. Kienholz $z^{6}$ utilizando fontes orgânicas de zinco na alimentação de aves com idade avançada, 142 semanas de idade no início do experimento, e sofrendo estresse devido ao nível baixo de cálcio na dieta observou melhora na produção e nos parâmetros de qualidade de ovo.

Paik $^{7}$ avaliando o uso de zinco metionina, cobre metionina e manganês metionina, isoladamente ou em combinação, observou que houve melhora na produção das aves que receberam o cobre metionina isolado, e a combinação dos três minerais, essa melhora não foi observada para o tratamento com o zinco metionina isolado e para o tratamento onde houve a combinação do cobre com o zinco orgânico.

A literatura ainda é muito controversa a respeito do uso dos minerais quelatados, portanto estudos devem ser realizados com o intuito de avaliar a resposta das aves aos minerais quelatados em relação aos minerais inorgânicos, bem como estudos que avaliem a estabilidade da molécula no trato digestivo das aves e a sua biodisponibilidade. Diante do exposto, este trabalho teve por objetivo avaliar índices de produtividade de poedeiras comerciais recebendo suplementação com fontes orgânicas e inorgânicas de zinco, cobre, manganês, selênio, ferro e iodo.

\section{Materiais e métodos}

O experimento foi realizado no setor de avicultura da Faculdade de Medicina Veterinária e Zootecnia da Universidade de São Paulo, sediado em Pirassununga (São Paulo), no período de março a junho de 2.003. Foram utilizadas 672 aves da linhagem Isa Babcock B-300 com idade de 48 semanas no inicio do experimento. As aves foram alojadas duas a duas em gaiolas de arame galvanizado, medindo $0,25 \times 0,40 \times 0,40 \mathrm{~m}$, dispostas em linha e providas de comedouro de chapa galvanizada e bebedouros tipo "nipple". O período experimental foi de 12 semanas, o que correspondeu de 48 a 60 semanas de idade das aves.

As aves foram pesadas no dia do início de cada fase do experimento, agrupadas conforme o peso, e então, distribuídas aleatoriamente aos tratamentos. A unidade experimental constou de 14 aves alojadas em 7 gaiolas adjacentes, com 6 repetições. O programa de iluminação artificial foi mantido constante durante todo o período experimental, de modo a fornecer 17 horas de luz diariamente.

O delineamento experimental utilizado foi o inteiramente casualizado, constituído de 8 tratamentos com seis repetições de 14 aves por parcela. Os tratamentos foram os seguintes: um grupo controle com micro minerais inorgânicos (manganês, zinco, selênio, iodo, cobre e ferro); um grupo com todos estes micro minerais orgânicos e mais 6 grupos onde cada mineral testado foi isoladamente substituído pela sua forma orgânica, conforme o esquema a seguir:

\section{Tratamentos Experimentais:}

Tratamento A - Controle - Todos micro minerais na forma inorgânica

Tratamento B - Todos micro minerais inorgânicos, sendo o Mn na forma orgânica

Tratamento C - Todos micro minerais inorgânicos, sendo o $\mathrm{Zn}$ na forma orgânica 
Tratamento D - Todos micro minerais inorgânicos, sendo o I na forma orgânica

Tratamento E - Todos micro minerais inorgânicos, sendo o Se na forma orgânica

Tratamento F - Todos micro minerais inorgânicos, sendo o $\mathrm{Cu}$ na forma orgânica

Tratamento G - Todos os micro minerais na forma orgânica

Tratamento H - Todos micro minerais inorgânicos, sendo o Fe na forma orgânica

As aves receberam nos períodos da $48^{a}$ a $60^{a}$ semanas de idade, uma mesma dieta para postura, a base de milho e soja, e que foi formulada para satisfazer as recomendações do National Research
Council ${ }^{8}$, e está relacionada na tabela $1 . \mathrm{O}$ alimento e a água foram fornecidos à vontade ao longo do período experimental. Os níveis dos minerais utilizados nos tratamentos também seguiram as recomendações do National Research Council ${ }^{8}$. Foram preparados 8 premixes minerais sendo que os mesmos apresentavam níveis iguais de minerais.

Os efeitos dos tratamentos foram medidos através de parâmetros de desempenho. A avaliação abrangeu: Consumo de Ração (g/ave/dia), Produção de Ovos (\%), Massa de Ovos (g/ave/dia), e Conversão Alimentar (kg de ração consumida/dúzias de ovos produzidos).

Os resultados obtidos foram analisados através do programa computacional Statistical Analysis System,

Tabela 1 - Composição da ração basal (utilizada para a preparação de todas as dietas experimentais). Pirassununga, 2003

\begin{tabular}{|c|c|}
\hline INGREDIENTES & $\%$ \\
\hline Milho & 57,48 \\
\hline Farelo de Soja & 28,31 \\
\hline Óleo vegetal & 2,54 \\
\hline Sal & 0,34 \\
\hline Calcário 38 \% & 8,91 \\
\hline Fosfato bicálcico & 1,93 \\
\hline Cloreto de Colina $50 \%$ & 0,05 \\
\hline DL - Metionina $99 \%$ & 0,14 \\
\hline Premix Vitamínico $\left.\quad{ }^{*}\right)$ & 0,10 \\
\hline Premix Mineral $(* *)$ & 0,20 \\
\hline Total & 100,00 \\
\hline \multicolumn{2}{|l|}{ COMPOSIÇÃO ESTIMADA } \\
\hline Energia Metabolizável, Kcal / kg & 2840 \\
\hline Proteína, \% & 18,00 \\
\hline Metionina, $\%$ & 0,43 \\
\hline Metionina + Cistina, $\%$ & 0,73 \\
\hline Cálcio, \% & 3,95 \\
\hline Fósforo Total, \% & 0,69 \\
\hline Fósforo Disponível, \% & 0,46 \\
\hline
\end{tabular}

(*) Premix vitamínico fornece (por kg de dieta): vitamina A, 7000 Ul; vitamina D3, 2000 Ul; vitamina E, 5 mg; vitamina K, 1,6 mg; tiamina, 0,7 mg. riboflavina, $3 \mathrm{mg}$; piridoxina, 2,5 mg; vitamina B12, 8 mcg; ácido nicotinico, 20 mg; pantotenato de cálcio, 5 mg; ácido fólico, 0,250mg; biotina, $0,1 \mathrm{mg}$.

(**) Premix mineral fornece (por kg de dieta): Manganês: 30mg, Zinco: 50 mg, lodo: 0,30 mg, Selênio: 0,10 mg, Cobre: 6 mg, Ferro: 45 mg. 
sendo anteriormente verificada a normalidade dos resíduos pelo Teste de Shapiro-Wilk (PROC UNIVARIATE) e as variâncias comparadas pelo Teste de Hartley Os dados originais ou transformados, quando este último procedimento foi necessário, foram submetidos à análise de variância que separou como causas de variação efeito de forma de apresentação do mineral. Os efeitos das variáveis de produção de ovos foram separados através do teste de separação de médias (teste de Tukey), enquanto que na presença de interação forma do mineral*tempo, optouse pelo procedimento General Linear Model (PROC GLM do SAS). Foi utilizado um nível de significância de 5\% para todos os testes realizados.

\section{Resultados e Discussão}

As médias de consumo de ração ( $\mathrm{g} /$ ave/dia), produção de ovos (\% ave/dia), Massa de ovos (g/ave/dia), e Conversão Alimentar (kg ração/dúzias de ovos), são apresentadas respectivamente nas tabelas 2 , 3, 4, e 5. A análise foi feita comparando-se os dados obtidos em 3 períodos experimentais consecutivos (P 1, P 2 e P 3), cada um com 28 dias de duração.

A analise das médias de desempenho obtidas para os diferentes períodos estudados apenas revelou diferença significativa de fontes de minerais para o parâmetro massa de ovos, sendo que o tratamento no qual as aves receberam somente o ferro na forma quelatada, apresentou menor valor para esta característica $(p<0,05)$, quando comparado com todos os outros tratamentos. A diferença foi observada na ultima avaliação que foi efetuada quando as aves apresentavam 60 semanas de idade (P 3). A analise efetuada não detectou efeitos da suplementação isolada dos minerais testados na sua forma orgânica com as formas totalmente orgânica e totalmente inorgânica, para os demais parâmetros de desempenho produtivo considerados, pois o consumo de ração não foi alterado pelos tratamentos empregados dentro de um mesmo ciclo de postura, para os períodos analisados e os valores médios obtidos para a percentagem de postura não apresentaram diferenças estatísticas para nenhum dos períodos estudados. Também não foi observada melhora na conversão alimentar ( $\mathrm{kg} / \mathrm{dz}$ ovo) para nenhum dos tratamentos nos quais se empregaram minerais orgânicos, quando comparados com o tratamento inorgânico, durante os diferentes períodos de experimentação.

No presente estudo, para os parâmetros de desempenho: consumo de ração, percentagem de produção de ovos, e conversão alimentar, não foram constatadas diferenças significativas em nenhum dos períodos estudados. Desta maneira, as diferentes fontes de minerais empregadas mostraram-se adequadas para maximizar estes parâmetros produtivos, de forma que a fonte não teve efeito adicional. Levando-se em consideração a adição das diferentes fontes minerais para rações de poedeiras comerciais, temos que os dados do presente experimento concordam com os obtidos por alguns autores, como: Kienholz ${ }^{6}$; Lundeen ${ }^{10}$ e Paik ${ }^{7}$ que não encontraram diferenças entre o uso de minerais orgânicos e minerais inorgânicos.

A ausência de efeito das diferentes fontes minerais também foi observada por Dale e Stong ${ }^{11}$ que testaram a eficácia de um produto comercial descrito como a combinação de proteinato com microminerais, fornecido para poedeiras com 80 semanas de idade. Durante um período de avaliação de 5 semanas, o produto não teve efeito sobre a produção de ovos e qualidade da casca.

$\mathrm{Na}$ avaliação do parâmetro Massa de ovos foi observado no presente experimento uma diferença significativa ( $p<0,05)$, e apenas as aves que receberam o ferro quelatado apresentaram menor valor que o observado nos demais tratamentos, sendo que esta diferença foi detectada apenas no último período experimental. 
Tabela 2-Consumo de ração (g/ave/dia): Efeitos dediferentes fontes de minerais, conformea idade das aves, nos períodos estudados. Pirassununga, 2003

\begin{tabular}{ccccc}
\hline \multirow{2}{*}{ Tratamento } & \multicolumn{3}{c}{ Ciclos de Postura de 28 dias } & Média \\
\hline A & 103,29 & 104,32 & 105,71 & 104,44 \\
B & 103,36 & 107,04 & 104,47 & 104,96 \\
C & 101,79 & 105,99 & 104,77 & 104,18 \\
D & 102,29 & 103,82 & 104,65 & 103,59 \\
E & 105,18 & 107,72 & 105,34 & 106,08 \\
F & 103,64 & 105,77 & 106,34 & 105,25 \\
G & 104,32 & 105,65 & 105,48 & 105,15 \\
H & 103,02 & 104,57 & 103,05 & 103,55 \\
\hline MÉDIA & 103,36 & 105,61 & 104,97 & 104,65 \\
EPM** & 1,0529 & 1,0529 & 1,0529 & \\
PROB & 0,4136 & 0,1408 & 0,5201 & \\
\hline
\end{tabular}

$(* *)$ EPM = erro padrão da média

Médias de tratamentos seguidas por letras diferentes (nas colunas) diferem significativamente pelo Teste de t-Student $(\mathrm{a}=5 \%)$

Paik $^{7}$ observou uma piora na massa de ovos para o tratamento contendo o cobre na forma orgânica; os demais tratamentos não se diferenciaram do tratamento controle; o qual era inorgânico. $\mathrm{O}$ autor não encontrou diferenças no tratamento com o ferro orgânico, desta forma não concordando com os resultados obtidos no presente experimento.

$O$ ferro na forma quelatada deve ser mais bem estudado, tendo em vista que a absorção do mesmo depende de um processo ativo do qual participam proteínas transportadoras na membrana das células intestinais, e que a reutilização de ferro pelo organismo é um processo bem eficiente. Paik ${ }^{7}$ observou que o uso de formas quelatadas de ferro aumenta em até $20 \%$ o conteúdo de ferro da gema do ovo, e segundo este autor, o fator mencionado poderia ser uma boa justificativa para o uso dos quelatos.

Kienholz $z^{6}$ demonstrou que o uso de zinco quelatado para aves com estresse devido ao baixo nível de cálcio na dieta (3\%), provocou a manutenção do tamanho do ovo quando comparado com o tratamento com mineral inorgânico que ocasionou uma redução no período do estresse. Os demais parâmetros não foram alterados pelos tratamentos. Estes resultados são confirmados por Lundeen ${ }^{10} \mathrm{em}$ um trabalho semelhante. $\mathrm{O}$ autor concluiu que os quelatos podem ser considerados como sendo uma opção ao desempenho produtivo. Este fato poderia talvez ser explicado pela grande quantidade de várias moléculas quelatadas existentes e suas diferenças na biodisponibilidade e estabilidade, bem como no seu meta-bolismo.

Os níveis dos minerais utilizados neste experimento seguiram as recomendações do National Research Council ${ }^{8}$ que é feita em base dos minerais inorgânicos, deve se considerar que as fontes quelatadas apresentam uma melhor biodisponibilidade e, portanto devendo ter uma menor inclusão na dieta, mas com o mesmo desempenho das fontes inorgânicas.

Atualmente na avicultura a preocupação com o meio ambiente é cada vez maior, principalmente quando se diz respeito ao mercado internacional, tendo em vista esse aspecto o uso dos minerais orgânicos podem reduzir a quantidade de mineral excretado pelas aves, devido a sua maior biodisponibilidade o que propicia que esses sejam incluídos em menores dosagens na dieta das aves. 
Tabela 3 - Percentagem de postura (\%): Efeitos de diferentes fontes de minerais, conforme a idade das aves, nos períodos de 28 dias estudados. Pirassununga, 2003

\begin{tabular}{ccccc}
\hline & \multicolumn{3}{c}{ Ciclos de Postura de 28 dias } & Média \\
\hline Tratamento & P 1 & P 2 & P 3 & 88,18 \\
A & 89,05 & 87,17 & 88,32 & 88,96 \\
B & 89,02 & 91,08 & 86,75 & 88,51 \\
C & 88,90 & 89,28 & 87,34 & 90,17 \\
D & 91,41 & 89,79 & 89,29 & 90,28 \\
E & 90,99 & 91,53 & 88,31 & 89,05 \\
F & 89,50 & 88,43 & 89,21 & 90,64 \\
G & 90,03 & 90,67 & 91,20 & 88,06 \\
H & 89,88 & 88,88 & 85,43 & 89,23 \\
MÉDIA & 89,85 & 89,60 & 88,23 & \\
EPM ** & 1.3449 & 1.3449 & 1.3449 & \\
PROB & 0,8449 & 0,3221 & 0,1166 &
\end{tabular}

$(* *)$ EPM $=$ Erro Padrão da Média

Médias de tratamentos seguidas por letras diferentes (nas colunas) diferem significativamente pelo Teste de t-Student $(\mathrm{a}=5 \%$ )

Tabela 4-Massa de ovos (g): Efeitos de diferentes fontes de minerais, conformea idade das aves, nos períodos de 28 dias estudados. Pirassununga, 2003

\begin{tabular}{ccccc}
\hline & \multicolumn{3}{c}{ Ciclos de Postura de 28 dias } & Média \\
\hline Tratamento & P 1 & P 2 & P 3 & 56,18 \\
A & 55,87 & 55,28 & $57,39^{\mathrm{a}}$ & 56,44 \\
B & 55,87 & 58,19 & $55,24^{\mathrm{a}}$ & 56,34 \\
C & 55,79 & 56,81 & $56,39^{\mathrm{a}}$ & 56,86 \\
D & 57,15 & 56,56 & $56,87^{\mathrm{a}}$ & 57,82 \\
E & 57,76 & 58,47 & $57,20^{\mathrm{a}}$ & 56,91 \\
F & 56,23 & 56,67 & $57,82^{\mathrm{a}}$ & 57,94 \\
G & 57,11 & 57,64 & $59,06^{\mathrm{a}}$ & 55,51 \\
H & 55,99 & 56,10 & $54,43^{\mathrm{b}}$ & 56,75 \\
\hline Média geral & 56,47 & 56,96 & 56,80 & \\
EPM ${ }^{* *}$ & 0,9247 & 0,9247 & 0,9247 & \\
P $=\left(^{*}\right)$ & 0,7006 & 0,2386 & 0,0229 & \\
\hline
\end{tabular}

${ }^{*}$ ) nível de significância do teste que compara as médias dos tratamentos em cada ciclo.

$(* *)$ EPM $=$ Erro Padrão da Média.

Médias de tratamentos seguidas por letras diferentes (nas colunas) diferem significativamentepelo Teste de t-Student $(\mathrm{a}=5 \%)$

\section{Conclusões}

Os resultados do presente estudo de avaliação da suplementação mineral na forma orgânica quando comparada com a forma inorgânica sobre o desempenho produtivo de poedeiras no período de 48 a 60 semanas de idade permitem concluir que: - O uso de formas orgânicas dos minerais: $\mathrm{Mn}, \mathrm{Zn}, \mathrm{I}, \mathrm{Se}, \mathrm{Cu}$ e Fe, isolada ou conjuntamente, não trouxe benefícios aos parâmetros de produção. Partindo-se da 
Tabela 5 - Conversão Alimentar (Kg/dúzias): Efeitos de diferentes fontes de minerais, conformea idade das aves, nos períodos de 28 dias estudados. Pirassununga, 2003

\begin{tabular}{ccccc}
\hline & \multicolumn{3}{c}{ Ciclos de Postura de 28 dias } & Média \\
\hline Tratamento & P 1 & P 2 & P 3 & 1,42 \\
A & 1,39 & 1,44 & 1,43 & 1,42 \\
B & 1,39 & 1,41 & 1,45 & 1,41 \\
C & 1,37 & 1,42 & 1,44 & 1,38 \\
D & 1,34 & 1,39 & 1,40 & 1,41 \\
E & 1,38 & 1,41 & 1,43 & 1,42 \\
F & 1,39 & 1,43 & 1,42 & 1,39 \\
G & 1,38 & 1,40 & 1,39 & 1,41 \\
H & 1,38 & 1,40 & 1,45 & 1,41 \\
MÉDIA & 1,38 & 1,41 & 1,43 & \\
EPM $\left(^{*}\right)$ & 0.01793 & 0.01793 & 0.01793 & 0,4101 \\
PROB & 0,4974 & 0,5740 & &
\end{tabular}

(*) $\mathrm{EPM}=$ Erro Padrão da Média

premissa de que estas fontes apresentam maior biodisponibilidade, o efeito de seu uso permanece controverso.

- A suplementação isolada de cada mineral orgânico estudado não mostrou melhor resultado que os tratamentos com os premixes totalmente orgânico e totalmente inorgânico.

\section{Effects of diet supplementation with organic trace minerals on production of laying hens}

\section{Abstract}

An experiment was conducted to study the effect of organic trace mineral sources supplementation on egg production of laying hens. It consisted of 8 treatments: one treatment with all trace minerals on inorganic form, one treatment with all minerals on organic form and 6 treatments where only one mineral was from organic origem. The trace minerals were: zinc, manganese, iodine, selenium, copper and iron. Corn and soybean meal basal diet was used. 672 Babcock B300 laying hens with 48 weeks were used. The production parameters analyzed were: Feed intake, egg production, egg mass and feed conversion. Data were summarized for 3 periods of 28 days each and analyzed throught SAS. The results for egg production were not different for the tree periods, except for egg mass witch was lowed in the organic iron treatment in the last period $(\mathrm{p}<0,05)$. The experiment results suggest that the supplementation with organic minerals did not improve the egg production for laying hens between $48-60$ weeks of age, when it was compared with inorganic mineral supplementation.
Key words:

Layers.

Egg (production), Trace mineral. Chelated,. Performance. 


\section{Referências}

1 ROLAND, D. A. Eggshell breakage: incidence and economic impact. Poultry Science, v. 67, p. 1801-1803, 1998.

2 LEESON, S.; SUMMERS, J. D. Nutrition of the chicken. 4. ed. Guelph, Ontario: University Books, 2001. 591 p.

3 REDDY, A. B.; DWIVED J. N.; ASHMEAD, A. D. Mineral chelation generates profit. Misset-World Poultry, v. 8, p. 13-15, 1992.

4 MORENG, R. E. Dietary zinc methionine effect on shell quality of hens drinking saline water. Poultry Science, v. 71, p. 1163-1167, 1992.

5 BALNAVE, D.; ZHANG, D. Responses of laying hens on saline drinking water to dietary supplementation with various zinc compouds. Poultry Science, v. 72, p. 603-606, 1993.

6 KIENHOLZ, E. W. Zinc methionine for stressed laying hens. Poultry Science, v. 71, p. 829-832, 1992.

7 PAIK, I. Application of chelated minerals in animal production. Asian-Australasian Journal of Animal Sciences, v. 14, p. 191-198, 2001.

8 NATIONAL RESEARCH CONCIL. Nutrient requeriment of poultry. 9. ed., Washington, 1994. 156p.

9 OTT, R. L. An introduction to statistical methods and data analysis. 3. ed. Boston: PWS-Kent, 1988. 110 p.

10 LUNDEEN, T. Mineral proteinates may have positive effect on shell quality. Feedstuffs, v. 73, n. 14, p. 1015,2001

11 - DALE, N.; STONG, C. F. Inability to demonstrate na effect of eggshell 49 on shell quality in older laying hens. Applied Poultry Science, v. 7, p. 219-224, 1998.

12 BRANTON, S. L. et al. Fatty liver-hemorrhagic syndrome observed in commercial layers fed diets containing chelated minerals. Avian Diseases, v. 39, p. 631-635, 1995. 